\title{
Correlation between CD64 and PCT levels in cerebrospinal fluid and degree of hearing impairment sequelae in neonates with purulent meningitis
}

\author{
CUI LIU ${ }^{1,2}$ and DONGCHI ZHAO ${ }^{1}$ \\ ${ }^{1}$ Department of Pediatrics, Zhongnan Hospital of Wuhan University, Wuhan, Hubei 430071; \\ ${ }^{2}$ Hubei University of Medicine, Shiyan, Hubei 442000, P.R. China
}

Received May 15, 2017; Accepted September 14, 2017

DOI: $10.3892 /$ etm.2017.5273

\begin{abstract}
This study investigated the possible correlation between the degree of hearing impairment caused by neonatal purulent meningitis and the levels of CD64 and PCT in cerebrospinal fluid of patients, and assessed the prognostic value of such levels. We recorded data from 156 cases of neonatal purulent meningitis retrospectively. All the patients received brainstem response audiometry, and cerebrospinal fluid samples were collected within the first day after admission through lumbar puncture. Flow cytometry was used to detect CD64 levels and enzyme-linked fluorescent assay was used to detect PCT levels. The children with hearing impairment were followed up for 1 year and brainstem response audiometry was performed again in them. We found that $43.59 \%$ of the children showed different degrees of hearing impairment, and $55 \%$ of them did not fully recover. The levels of PCT and CD64 in cerebrospinal fluid of children with hearing impairment were significantly higher than those of children with normal hearing $(\mathrm{P}<0.01)$. The levels of PCT and CD64 in mild, moderate and severe hearing impaired children increased gradually with higher degrees of impairment, and the differences between groups were significant $(\mathrm{P}<0.01)$. During the follow-up, it was found that the levels of PCT and CD64 in children correlated well with the degree of hearing recovery, and the differences between groups were significant $(\mathrm{P}<0.01)$. In our study, approximately $1 / 4$ children with purulent meningitis showed long-term hearing impairment. Based on our analyses, the levels of CD64 and PCT in cerebrospinal fluid can be used to predict the degree and long-term prognosis of hearing impairment caused by purulent meningitis in children.
\end{abstract}

Correspondence to: Dr Dongchi Zhao, Department of Pediatrics, Zhongnan Hospital of Wuhan University, 169 East Lake Road, Wuchang, Wuhan, Hubei 430071, P.R. China

E-mail: dongchi_zhao@hotmail.com

Key words: purulent meningitis, cerebrospinal fluid, hearing impairment, CD64, PCT

\section{Introduction}

Purulent meningitis is a serious central nervous system infection caused by pyogenic bacteria $(1,2)$, and neonatal purulent meningitis refers to its occurrence within the first 4 weeks after birth. Common etiological agents include Group B streptococci, Escherichia coli, Staphylococcus aureus and Listeria monocytogenes $(3,4)$. The morbidity rate in live-born infants for neonatal purulent meningitis is approximately $0.06-1 \%$, and in premature infants is up to $3 \%(5,6)$. The disability and mortality rates in those affected are both high: In developed countries, the disability rate reaches $20-50 \%$, and the mortality rate is approximately 10-15\% (7-10). While in developing countries, the mortality rate is as high as $40-58 \%$ (11). Recent medical advancements are likely to reduce the morbidity and mortality rates for neonatal purulent meningitis, but the disability rate in neonates is still going to remain high. Hearing impairment, which is the most common type of disability caused by the infection (9), can seriously affect a child's development. $\mathrm{CD}^{+} 4^{+}$neutrophils and procalcitonin (PCT) are specific markers for bacterial infection that are widely used in the diagnosis of infectious diseases such as neonatal pneumonia, sepsis and others. In this study, we retrospectively recorded the levels of CD64 and PCT in cerebrospinal fluid samples of 156 cases of neonatal purulent meningitis, also auditory tests and clinical follow-ups were performed to detect degrees of hearing impairment left as sequelae. Statistical analyses were performed to explore the correlation of CD64 and PCT levels with hearing impairment and their prognostic values.

\section{Patients and methods}

Clinical data. Clinical data of 156 cases of neonatal purulent meningitis were recorded during a period from April 2012 to April 2016 in Zhongnan Hospital. There were 91 males and 65 females. All the patients received brainstem response audiometry tests. During the cases review each case was assigned either to a normal hearing or an impaired hearing group. The patients in the impaired hearing group were followed up for 1 year. During follow-up, 1 patient died and 7 patients failed to be present. The family members of all the patients signed informed consents. The study was approved by the Ethics 
Table I. Hearing conditions of the patients on the day of admission.

\begin{tabular}{lccc}
\hline Patient groups & $\begin{array}{c}\text { Mild hearing } \\
\text { impairment }\end{array}$ & $\begin{array}{c}\text { Moderate hearing } \\
\text { impairment }\end{array}$ & $\begin{array}{c}\text { Severe hearing } \\
\text { impairment }\end{array}$ \\
\hline Normal hearing group $(\mathrm{n}=88)$ & - & - & - \\
Hearing impairment group $(\mathrm{n}=68)$ & 18 & 23 & 27 \\
Patients who finished follow-up $(\mathrm{n}=60)$ & 16 & 21 & 23 \\
\hline
\end{tabular}

Committee of Zhongnan Hospital of Wuhan University (Protocol no. 2015019).

Experimental methods. Diagnostic and exclusion criteria for all the children in the study were taken from the fourth edition of 'Practical Pediatrics'. All children received lumbar puncture for cerebrospinal fluid (CSF) examination and the results of the test included at least one of the following eight possibilities for the CSF routine test: The CSF appeared turbid after smear or culture. The CSF pressure was higher than 2.94-7.84 kPa. The CSF white blood cell count was higher than $29 \times 10^{6} / 1$ in premature infants, higher than $32 \times 10^{6} / 1$ in term infants and higher than $10 \times 10^{6} / 1$ in patients at 1 week after birth. More than $57-61 \%$ of the cells were multinucleated cells. The protein content was higher than $0.65-1.5 \mathrm{~g} / 1$ in premature infants and higher than 0.1-1.7 g/l in term infants. The Pan test gave ++ or +++ . The lactate dehydrogenase was often higher than $1000 \mathrm{U} / 1$. In addition, the glucose levels were often lower than 1.1-2.2 mmol/l. For CSF cell counts, the proportions of white blood cells and erythrocytes were significantly higher than those in blood. CSF smear and cultures were negative. For patients with abnormalities in hearing screening, a family history of deafness, neonatal hyperbilirubinemia, intrauterine infection history, perinatal hypoxia asphyxia history, congenital brain development and other abnormalities were excluded.

Audiological examination was performed using the evoked potentials instrument (Chartr-EP) at the ENT Department of Zhongnan Hospital of Wuhan University. The test was performed either during sleep or after a phenobarbital injection. The test was performed in a soundproof audiometric test room. The recording electrode was affixed to the cranial $\mathrm{CZ}$ site, and the reference electrode was attached to the ipsilateral mastoid bone prominence. After clearing foreign bodies in the ear, appropriate earplugs were used. One ear was stimulated with sparse polar burst sound, and the other ear was treated with flat noise. The data were recorded at the intensity of $70 \mathrm{~dB}$. Each ear was tested at least twice. The threshold for audiological examination was set with the sound stimulation from an intensity of $30 \mathrm{~dB}$, increasing gradually to a maximum stimulation up to $97 \mathrm{~dB}$. The high frequency hearing threshold was defined as the sound intensity that can be recognized up to the $\mathrm{V}$ wave. The normal high frequency hearing threshold of this experiment was $30 \mathrm{~dB}$. According to audiology standards, less than $40 \mathrm{~dB}$ was classified as mild hearing impairment, $41-55 \mathrm{~dB}$ indicated a moderate hearing impairment, 56-70 dB indicated a moderate to severe hearing impairment, 71-90 dB indicated a severe hearing impairment and higher than $91 \mathrm{~dB}$ indicated an extremely severe hearing impairment.
Detection of CD64 and PCT. CSF was collected through lumbar puncture within the first day after hospitalization for a CSF routine test, and biochemical and virological tests. Additionally, a portion of the fluid was used for CD64 and PCT detection. A flow cytometer (Cytomics FC500, Berea, $\mathrm{OH}, \mathrm{USA}$ ) was used to detect the CD64 content. The detection of PCT was performed through enzyme-linked fluorescence analysis using a ELISA kit (Biomerica, Irvine, CA, USA), and a PCT $\geq 0.5 \mathrm{ng} / \mathrm{ml}$ was considered to be positive.

Statistical analysis. The statistical analysis was performed using SPSS 19.0 statistical software (SPSS Inc., Chicago, IL, USA). Measurement data were expressed as mean $\pm \mathrm{SD}$, the comparisons between two groups were performed using independent sample t-tests, and the comparisons among three groups were performed by one-way ANOVA. Graded data were expressed as counts, and the Kruskal-Wallis $\mathrm{H}$ test was used for the comparisons among more than 2 groups. $\mathrm{P}<0.05$ was considered to indicate a statistically significant difference.

\section{Results}

Hearing conditions of the patients on the day of admission. This study includes the analysis of data from 156 cases of neonatal purulent meningitis. The data from patients were divided into two groups, a normal hearing and a hearing impairment group (68 patients accounting for $43.59 \%$ of all the patients). At the same time, dta from children with hearing impairment were subdivided into three groups according to mild (18 cases), moderate ( 23 cases) or severe (27 cases) hearing impairment degree. All children with abnormal hearing were followed up for a minimum of six months and up to three years. During the follow-up, 1 patient died and 7 failed to show up to complete the follow-ups. Among the 60 patients who finished the follow-ups, 16 showed mild hearing impairment, 21 moderate hearing impairment, and 23 severe hearing impairment on the day of admission (Table I).

Comparison of hearing conditions of patients during follow-up. Among the 16 patients who presented mild hearing impairment at the time of admission, 13 completely recovered, 2 showed partially recovery, and 1 did not recover at all. Among the 21 patients with moderate hearing impairment at the time of admission, 10 recovered completely to normal hearing conditions, 8 recovered partially, and 3 did not recover. Finally, among the 23 patients showing severe hearing impairment at the time of admission, 4 recovered completely, 6 recovered partially, and 13 did not recover ( 2 had even worse conditions at the time of the follow-ups). The Kruskal-Wallis $\mathrm{H}$ test was used 
Table II. Comparison of hearing conditions of patients during follow-up.

\begin{tabular}{lccc}
\hline Patient groups & Complete recovery & Partial recovery & No recovery \\
\hline Mild hearing impairment $(\mathrm{n}=16)$ & 13 & 2 & 1 \\
Moderate hearing impairment $(\mathrm{n}=21)$ & 10 & 8 & 3 \\
Severe mild hearing impairment $(\mathrm{n}=23)$ & 4 & 6 & 13 \\
\hline
\end{tabular}

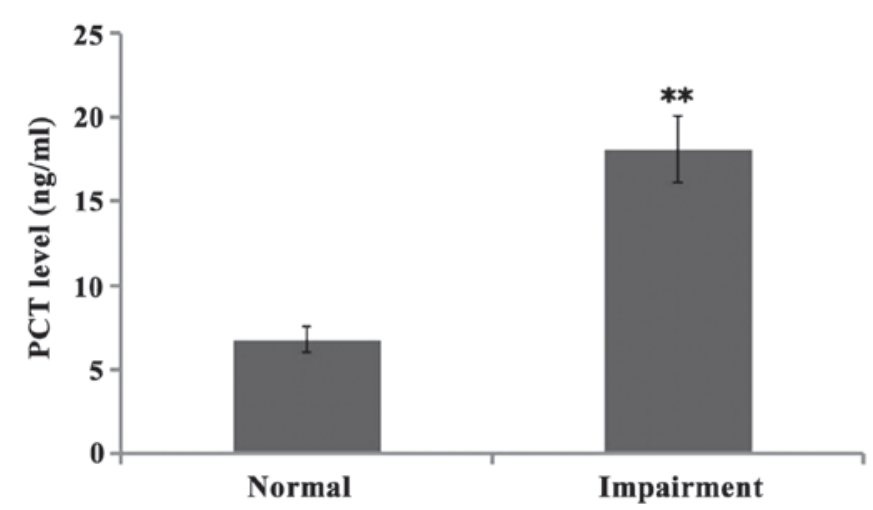

Figure 1. Comparison of PCT levels between patients with normal hearing and hearing impairment by enzyme-linked fluorescence. ${ }^{* *} \mathrm{P}<0.01$ compared with normal hearing group.

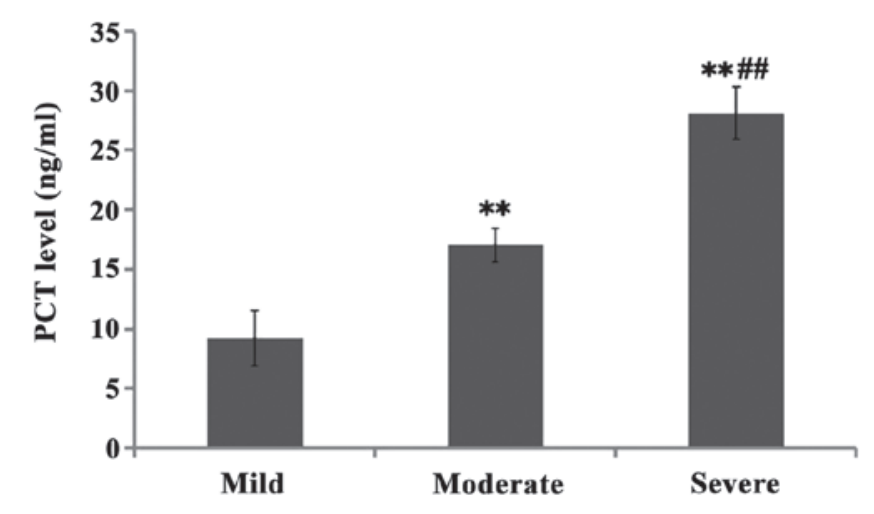

Figure 2. Comparison of PCT levels among patients with different degrees of hearing impairment by enzyme-linked fluorescence tests. ${ }^{* *} \mathrm{P}<0.01$ compared with the mild hearing impairment group; ${ }^{\#} \mathrm{P}<0.01$ compared with the moderate hearing impairment group.

for comparison among the three groups with different degrees of hearing impairment, and the results showed significant differences among the three groups $\left(\chi^{2}=18.315, \mathrm{P}<0.01\right)$ (Table II).

Comparison of PCT levels in CSF between patients. The levels of PCT in CSF of children with normal hearing and hearing impairment were both high when compared to the standard normal level, namely $6.78 \pm 0.770 .5 \mathrm{ng} / \mathrm{ml}$ and $18.12 \pm 1.980 .5 \mathrm{ng} / \mathrm{ml}$, respectively. There was significant difference between the two groups $(\mathrm{P}<0.01)$. Further comparison of PCT levels in cerebrospinal fluid of patients with varying degrees of hearing impairment showed that the levels of PCT increased gradually with the severity of the impairment, and the differences between groups were significant $(\mathrm{P}<0.01)$. Further comparisons showed the levels of PCT levels in cerebrospinal

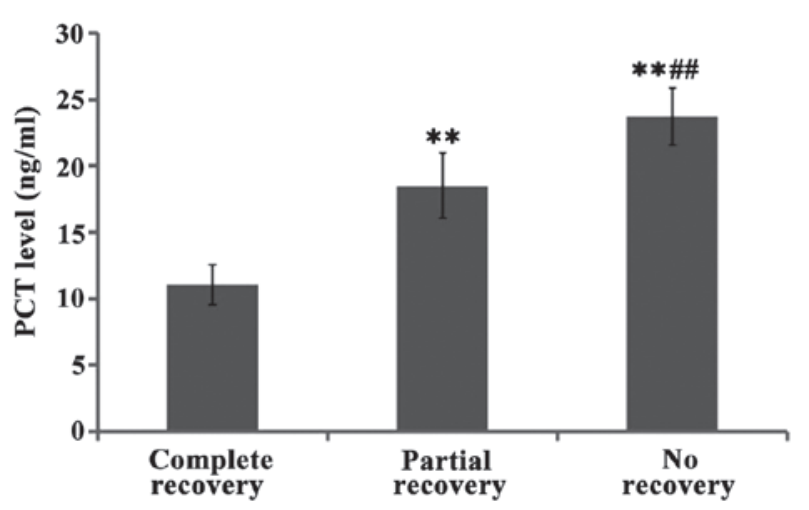

Figure 3. The comparison of PCT levels between patients with different degrees of hearing recovery. Enzyme-linked fluorescence tests showed that the levels of PCT in patients with partial recovery and non-recovery were significantly higher than that in patients with complete recovery $(\mathrm{P}<0.01)$, and the level of PCT in patients with non-recovery was significantly higher than that in patients with partial recovery $(\mathrm{P}<0.01) .{ }^{* *} \mathrm{P}<0.01$ compared with the patients with complete recovery; ${ }^{\#} \mathrm{P}<0.01$ compared with the patients with partial recovery.

fluid of patients with full recovery were lower than those of patients with partial recovery, and even lower than those of patients who did not recover at all, and the differences between groups were significant $(\mathrm{P}<0.01)$ (Figs. 1-3).

Comparison of CD64 levels CSF between patients. The levels of CD64 in cerebrospinal fluid of normal hearing and hearing impairment group patients were 1584.05 \pm 351.12 and $2208.79 \pm 427.30$, respectively, and there was significant difference between the two groups $(\mathrm{P}<0.01)$. Further comparison of CD64 levels showed the levels increased gradually from mild, to moderate and to severe hearing impairment groups, and the differences between groups were significant $(\mathrm{P}<0.01)$. Furthermore, the levels of CD64 in children with complete hearing recovery were lower than the levels in those who recovered partially and even lower than those who did not recover at all (with significant differences $\mathrm{P}<0.01$ ) (Figs. 4-6).

\section{Discussion}

Neonatal purulent meningitis is a common central nervous system pediatric infection. It can leave serious sequelae, that negatively impact the development and life quality of patients. Even though medical advances have lowered the mortality due to purulent meningitis, its sequelae are still frequent (12). These include hemiparesia, epilepsy, hearing impairment, small head deformity, blindness and cerebral palsy (13). A follow-up study carried out in Wales and England found that the most common 


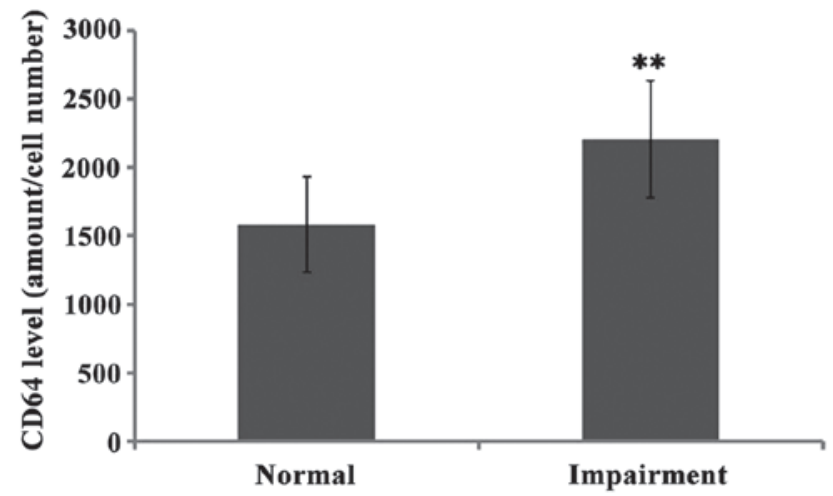

Figure 4. Comparison of CD64 levels between patients in the normal hearing and hearing impairment groups by flow cytometry. ${ }^{* *} \mathrm{P}<0.01$ compared with normal hearing group.

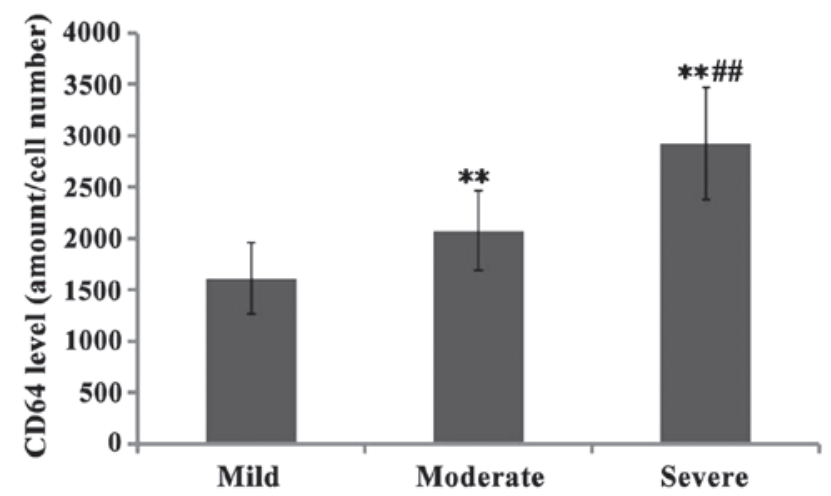

Figure 5. Comparison of CD64 levels among patients with different degree of hearing impairment. ${ }^{* *} \mathrm{P}<0.01$ compared with the mild hearing impairment group; ${ }^{\# \prime} \mathrm{P}<0.01$ compared with the moderate hearing impairment group.

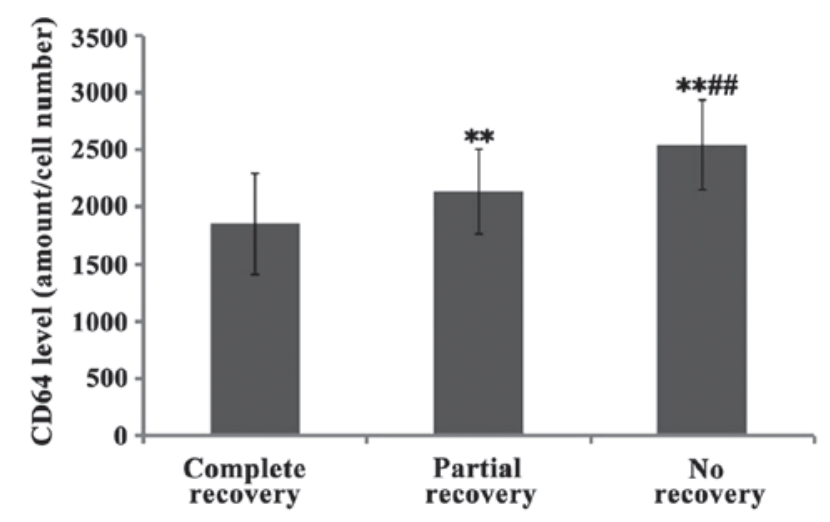

Figure 6. Comparison of PCT levels between patients with different degrees of hearing recovery. ${ }^{* *} \mathrm{P}<0.01$ compared with the patients with complete recovery; ${ }^{\# \#} \mathrm{P}<0.01$ compared with the patients with partial recovery.

sequelae of purulent meningitis is hearing impairment, and the second is language disorder, followed by vision disorder, and then behavioral and motor dysfunction (9). Another study also confirmed that hearing impairment is the most common sequelae, followed by mental retardation, dyskinesia and epilepsy (14). In this study, $43.59 \%$ of the patients (60) showed different degrees of hearing impairment, and $55 \%$ of the patients with hearing impairment failed to recover completely.
Therefore, we speculate that approximately $1 / 4$ of the patients who develop neonatal purulent meningitis will be left with long-term hearing impairment. We also found that the hearing recovery is related to the degree of the hearing impairment detected on the day of admission, whereas a higher degree of impairment increases the chances of incomplete recovery. It is possible that the inner ear hairy cells in patients with severe hearing impairment were damaged, resulting in irreversible changes in the microenvironment of the cochlea; supporting this notion is the fact that the patients with severe hearing impairment usually had congenital inner ear malformations. Mild hearing impairment, on the other hand, may possibly have been caused by otitis media tympanitis, which can easily be controlled. Further studies are still needed in order to understand the mechanisms that lead to life-long hearing impairment.

CD64, which is a member of the immunoglobulin superfamily, is a receptor of the Fc region of immunoglobulin IgG. Genes encoding CD64 are located on chromosome 1p13 and 1q21, and the molecular weight of the cognate protein is $72 \mathrm{kD}(15)$. There are three types of $\mathrm{IgG}$ Fc region receptors, namely CD64, CD32, and CD16, these receptors link cell and humoral immunities (16). CD64 is the only high affinity receptor for IgG. The expression of CD64 in the body is normally kept to low levels. After a bacterial infection, however, the body produces interferon, granulocyte colony stimulating factor, bacterial cell wall lipopolysaccharide and a series of neutrophil stimulating factors, causing increased expression of CD64 on the surface of neutrophils (17). A study found that the expression levels of CD64 differ in children and adults in different age groups (18), while the expression level does not significantly changed after viral infection, making it a useful marker for bacterial infection prediction. Yet another study showed that in children with septic shock, the expression levels of CD64 were positively correlated with the severity of infection, and higher expression levels indicated a more severe infection, suggesting that the expression level of CD64 can be used as an indicator of the severity of infection (19). In this study, the expression levels of CD64 in CSF in neonatal purulent meningitis patients were significantly increased; suggesting they can be used to assist in the diagnosis of neonatal purulent meningitis. Moreover, our study found that the level in CSF was related with the degree of hearing impairment of neonatal purulent meningitis patients, and higher expression levels of CD64 always came along with a higher degree of hearing impairment and lower degree of hearing recovery. This suggests that the expression level of CD64 can be used as a predictor of the degree of hearing impairment and prognosis for children with purulent meningitis.

PCT, a hormone activity-free glycoprotein, is a calcitonin pro-peptide. The PCT protein is composed of 16 amino acids with a total molecular weight of 13000 (20). Under physiological conditions, PCT is mainly produced and secreted by thyroid C cells. The normal content of PCT is very low in blood and undetectable in cerebrospinal fluid (20). After a bacterial infection, toxins secreted by bacteria can stimulate glandular cells to release a large amount of PCT, resulting in significantly increased PCT levels in blood and cerebrospinal fluid. Clinical studies have shown that PCT, WBC and CRP levels in blood and cerebrospinal fluid of patients with purulent meningitis 
get significantly increased $(21,22)$. Another study showed that the PCT level was significantly increased in the body $6 \mathrm{~h}$ after a bacterial infection in the body, and the increase of PCT appeared much earlier than the increase of CRP, indicating that PCT can be used as an early sensitive indicator of bacterial infection (23). This study found that the PCT levels in CSF of neonates with purulent meningitis were significantly increased. Furthermore, our study found that the PCT levels in CSF of the neonates with purulent meningitis correlate with the degree of hearing impairment, suggesting that the expression level of PCT can be used as a predictor of the degree of hearing impairment and prognosis for these patients.

In conclusion, this study found that approximately $1 / 4$ of children with purulent meningitis showed long-term hearing impairment. CD64 and PCT were highly expressed in the CSF of the patients. In addition, the expression levels were positively correlated with the degree of hearing impairment, whereas higher PCT levels were found in those cases with higher levels of hearing impairment and lower levels of recovery. Further clinical studies are still needed to understand the underlying mechanisms involved in the pathogenesis of neonatal purulent meningitis.

\section{References}

1. Kim KS: Pathogenesis of bacterial meningitis: From bacteraemia to neuronal injury. Nat Rev Neurosci 4: 376-385, 2003.

2. Mook-Kanamori BB, Geldhoff M, van der Poll $T$ and van de Beek D: Pathogenesis and pathophysiology of pneumococcal meningitis. Clin Microbiol Rev 24: 557-591, 2011.

3. Stoll BJ, Hansen NI, Sánchez PJ, Faix RG, Poindexter BB, Van Meurs KP, Bizzarro MJ, Goldberg RN, Frantz ID III, Hale EC, et al; Eunice Kennedy Shriver National Institute of Child Health and Human Development Neonatal Research Network: Early onset neonatal sepsis: The burden of group B Streptococcal and E. coli disease continues. Pediatrics 127 : 817-826, 2011

4. Ku LC, Boggess KA and Cohen-Wolkowiez M: Bacterial meningitis in infants. Clin Perinatol 42: 29-45, 2015.

5. Kim KS: Neonatal bacterial meningitis. Neoreviews 16 e535-e543, 2015.

6. May M, Daley AJ, Donath S and Isaacs D; Australasian Study Group for Neonatal Infections: Early onset neonatal meningitis in Australia and New Zealand, 1992-2002. Arch Dis Child Fetal Neonatal Ed 90: F324-F327, 2005.

7. Kaufman D, Zanelli S, Cantey JB and Sánchez PJ: Neonatal meningitis: current treatment options. In: Neurology: Neonatology Questions and Controversies. Perlman JMD (ed). 2nd edition. Elsevier, Amsterdam, pp181-201, 2012.
8. Holt DE, Halket S, de Louvois J and Harvey D: Neonatal meningitis in England and Wales: 10 years on. Arch Dis Child Fetal Neonatal Ed 84: F85-F89, 2001.

9. Reading R: Meningitis in infancy in England and Wales: Follow up at age 5 years. Child Care Health Dev 28: 533-536, 2002.

10. Stevens JP, Eames M, Kent A, Halket S, Holt D and Harvey D: Long term outcome of neonatal meningitis. Arch Dis Child Fetal Neonatal Ed 88: F179-F184, 2003

11. Furyk JS, Swann O and Molyneux E: Systematic review: Neonatal meningitis in the developing world. Trop Med Int Health 16: 672-679, 2011.

12. Grimwood K: Legacy of bacterial meningitis in infancy. Many children continue to suffer functionally important deficits. BMJ 323: 523-524, 2001.

13. Klinger G, Chin CN, Beyene J and Perlman M: Predicting the outcome of neonatal bacterial meningitis. Pediatrics 106: 477-482, 2000.

14. Bargui F, D'Agostino I, Mariani-Kurkdjian P, Alberti C, Doit C, Bellier N, Morin L, Galli Gibertini G, Smail A, Zanin A, et al: Factors influencing neurological outcome of children with bacterial meningitis at the emergency department. Eur J Pediatr 171: 1365-1371, 2012

15. Ravetch JV: Fc receptors: Rubor redux. Cell 78: 553-560, 1994.

16. Fossati G, Bucknall RC and Edwards SW: Fcgamma receptors in autoimmune diseases. Eur J Clin Invest 31: 821-831, 2001.

17. Du J, Li L, Dou Y, Li P, Chen R and Liu H: Diagnostic utility of neutrophil CD64 as a marker for early-onset sepsis in preterm neonates. PLoS One 9: e102647, 2014.

18. Chung JW, Park CJ, Cha CH, Cho YU, Jang S, Chi HS, Seo EJ, Lee JH, Lee JH, Lee KH, et al: A combination of CD15/CD10, CD64/CD33, CD16/CD13 or CD11b flow cytometric granulocyte panels is sensitive and specific for diagnosis of myelodysplastic syndrome. Ann Clin Lab Sci 42: 271-280, 2012.

19. Simonin-Le Jeune K, Le Jeune A, Jouneau S, Belleguic C, Roux PF, Jaguin M, Dimanche-Boitre MT, Lecureur V, Leclercq C, Desrues B, et al: Impaired functions of macrophage from cystic fibrosis patients: CD11b, TLR-5 decrease and sCD14, inflammatory cytokines increase. PLoS One 8: e75667, 2013.

20. Konstantinidis T, Cassimos D, Gioka T, Tsigalou C, Parasidis T, Alexandropoulou I, Nikolaidis C, Kampouromiti G, Constantinidis T, Chatzimichael A, et al: Can procalcitonin in cerebrospinal fluid be a diagnostic tool for meningitis? J Clin Lab Anal 29: 169-174, 2015

21. Abdelkader NA, Mahmoud WA and Saber SM: Serum procalcitonin in Egyptian patients with acute meningitis and a negative direct cerebrospinal fluid examination. J Infect Public Health 7: 106-113, 2014.

22. Choi HJ: Procalcitonin in diagnosis of post-operative bacterial meningitis: A promising but limited role. Infect Chemother 45: 346-348, 2013.

23. Viallon A, Desseigne N, Marjollet O, Birynczyk A, Belin M, Guyomarch S, Borg J, Pozetto B, Bertrand JC and Zeni F: Meningitis in adult patients with a negative direct cerebrospinal fluid examination: value of cytochemical markers for differential diagnosis. Crit Care 15: R136, 2011. 